University of Nebraska - Lincoln

DigitalCommons@University of Nebraska - Lincoln

USDA Wildlife Services - Staff Publications

U.S. Department of Agriculture: Animal and Plant Health Inspection Service

2020

\title{
Optimal spatial prioritization of control resources for elimination of invasive species under demographic uncertainty
}

Kim M. Pepin

USDA-APHIS, Fort Collins, kim.m.pepin@usda.gov

Timothy J. Smyser

USDA APHIS/Wildlife Services/NWRC, Timothy.J.Smyser@aphis.usda.gov

Amy J. Davis

USDA National Wildlife Research Center, Amy.J.Davis@usda.gov

Ryan S. Miller

USDA APHIS, Ryan.S.Miller@aphis.usda.gov

Sophie McKee

USDA/APHIS/WS National Wildlife Research Center \& Colorado State University, Eollow this andadditional works at: https://digitalcommons.unl.edu/icwdm_usdanwrc

Part of the Natural Resources and Conservation Commons, Natural Resources Management and

Policy Commons, Other Environmental Sciences Commons, Other Veterinary Medicine Commons, See next page for additional authors

Population Biology Commons, Terrestrial and Aquatic Ecology Commons, Veterinary Infectious Diseases

Commons, Veterinary Microbiology and Immunobiology Commons, Veterinary Preventive Medicine,

Epidemiology, and Public Health Commons, and the Zoology Commons

Pepin, Kim M.; Smyser, Timothy J.; Davis, Amy J.; Miller, Ryan S.; McKee, Sophie; Vercauteren, Kurt C.; Kendall, William; and Slootmaker, Chris, "Optimal spatial prioritization of control resources for elimination of invasive species under demographic uncertainty" (2020). USDA Wildlife Services - Staff Publications. 2371.

https://digitalcommons.unl.edu/icwdm_usdanwrc/2371

This Article is brought to you for free and open access by the U.S. Department of Agriculture: Animal and Plant Health Inspection Service at DigitalCommons@University of Nebraska - Lincoln. It has been accepted for inclusion in USDA Wildlife Services - Staff Publications by an authorized administrator of DigitalCommons@University of Nebraska - Lincoln. 


\section{Authors}

Kim M. Pepin, Timothy J. Smyser, Amy J. Davis, Ryan S. Miller, Sophie McKee, Kurt C. Vercauteren, William Kendall, and Chris Slootmaker 


\title{
Optimal spatial prioritization of control resources for elimination of invasive species under demographic uncertainty
}

\author{
Kim M. Pepin ID ${ }^{1,6}$ Timothy J. Smyser, ${ }^{1}$ Amy J. Davis (D) ${ }^{1}$ Ryan S. Miller, ${ }^{2}$ Sophie McKee, ${ }^{1,3}$ Kurt C. \\ VerCauteren, ${ }^{1}$ William Kendall, ${ }^{4}$ and Chris Slootmaker ${ }^{1,5}$ \\ ${ }^{1}$ National Wildlife Research Center, USDA-APHIS, Wildlife Services, 4101 Laporte Avenue, Fort Collins, Colorado80521USA \\ ${ }^{2}$ Centers for Epidemiology and Animal Health, USDA-APHIS, Veterinary Services, 2150 Centre Avenue, Fort Collins, \\ Colorado80526USA \\ ${ }^{3}$ Department of Economics, Colorado State University, Fort Collins, Colorado80523USA \\ ${ }^{4}$ Colorado Cooperative Fish and Wildlife Research Unit, U.S. Geological Survey, Colorado State University, 1484 Campus Delivery, \\ Fort Collins, Colorado80523USA \\ ${ }^{5}$ Mountain Data Group, 115 N. College Avenue, Suite 220, Fort Collins, Colorado80524USA
}

Citation: Pepin, K. M., T. J., Smyser, A. J., Davis, R. S., Miller, S., McKee, K. C., VerCauteren, W., Kendall, and C., Slootmaker. 2020. Optimal spatial prioritization of control resources for elimination of invasive species under demographic uncertainty. Ecological Applications 30(6):e02126. 10.1002/eap.2126

Abstract. Populations of invasive species often spread heterogeneously across a landscape, consisting of local populations that cluster in space but are connected by dispersal. A fundamental dilemma for invasive species control is how to optimally allocate limited fiscal resources across local populations. Theoretical work based on perfect knowledge of demographic connectivity suggests that targeting local populations from which migrants originate (sources) can be optimal. However, demographic processes such as abundance and dispersal can be highly uncertain, and the relationship between local population density and damage costs (damage function) is rarely known. We used a metapopulation model to understand how budget and uncertainty in abundance, connectivity, and the damage function, together impact return on investment (ROI) for optimal control strategies. Budget, observational uncertainty, and the damage function had strong effects on the optimal resource allocation strategy. Uncertainty in dispersal probability was the least important determinant of ROI. The damage function determined which resource prioritization strategy was optimal when connectivity was symmetric but not when it was asymmetric. When connectivity was asymmetric, prioritizing source populations had a higher ROI than allocating effort equally across local populations, regardless of the damage function, but uncertainty in connectivity structure and abundance reduced ROI of the optimal prioritization strategy by $57 \%$ on average depending on the control budget. With low budgets (monthly removal rate of $6.7 \%$ of population), there was little advantage to prioritizing resources, especially when connectivity was high or symmetric, and observational uncertainty had only minor effects on ROI. Allotting funding for improved monitoring appeared to be most important when budgets were moderate (monthly removal of $13-20 \%$ of the population). Our result showed that multiple sources of observational uncertainty should be considered concurrently for optimizing ROI. Accurate estimates of connectivity direction and abundance were more important than accurate estimates of dispersal rates. Developing cost-effective surveillance methods to reduce observational uncertainties, and quantitative frameworks for determining how resources should be spatially apportioned to multiple monitoring and control activities are important and challenging future directions for optimizing ROI for invasive species control programs.

Key words: bioeconomic model; connectivity; control; elimination; invasive species; metapopulation; optimal strategy; wild pig.

\section{INTRODUCTION}

The interaction of reproduction and dispersal processes determines the dynamics and persistence of spatially structured populations (Hanski 1998).

Manuscript received 21 May 2019; revised 16 January 2020; accepted 6 February 2020. Corresponding Editor: Erik J. Nelson.

${ }^{6}$ E-mail: kim.m.pepin@usda.gov
Reproduction provides new individuals that can disperse among local populations, promoting persistence in the global population. Rates and directions of dispersal in space define the "demographic connectivity" of a population. The concept of demographic connectivity underlies modern theory in conservation planning (Rudnick et al. 2012, van Nouhuys 2016). Conserving patches of habitat with higher levels of connectivity facilitates dispersal among local populations (Williams et al. 2004, Rudnick et al. 2012), which maximizes population 
growth rates and makes the entire population more resilient to extinction pressures (Hanski and Ovaskainen 2000, Williams et al. 2004, Hastings and Botsford 2006, Jacobi and Jonsson 2011).

An understanding of the importance of demographic connectivity is also used to optimize control of invasive species (Travis and Park 2004, Chades et al. 2011, Caplat et al. 2012, Glen et al. 2013, Baker 2017) and disease (Haydon et al. 2006, Chades et al. 2011). When control in spatially structured populations of invasive species is the objective, general guidelines for optimal prioritization have included to "target the most highly connected local populations" (Perry et al. 2017) and to "target local source populations" (Baker 2017) to minimize abundance and maximize extinction rates. These recommendations are consistent when considering a variety of connectivity structures, for example: managing upstream local populations when connectivity is directional, managing any local population and then its nearest neighbors when connectivity is a ring structure, and to start at one end and continue sequentially in bidirectional or linear connectivity structures (Chades et al. 2011). However, more complex recommendations can emerge when (1) one local population is highly connected and all others have only one connection, i.e., ring structures (Chades et al. 2011), (2) local populations exhibit source-sink dynamics (Travis and Park 2004), and (3) particular population growth (Caplat et al. 2014) and dispersal rates (Travis and Park 2004) are exhibited. Thus, the optimal strategy is affected by context-dependent demographic processes, including population growth rates and dispersal dynamics.

In practice, using knowledge of spatial population processes to manage populations ("connectivity-based management") requires estimates of demographic connectivity among local populations. Measures of demographic connectivity can be based on landscape features, or on estimates of dispersal behavior or gene flow between local populations (Baguette et al. 2012). Landscape-based estimates (e.g., least-cost path analysis) are useful for examining how processes such as landscape fragmentation and climate change might affect population connectivity and persistence, but usually neglect individual-level dispersal behavior (i.e., how animals actually travel through the landscape; Baguette et al. 2012). Estimates from tracking individual movement over time are ideal for understanding and predicting population persistence, but directly monitoring dispersal behavior for many individuals can be expensive in management applications. To address this gap, measures of gene flow have been used to infer demographic connectivity (Lowe and Allendorf 2010). However, models for estimating gene flow between local populations can require unrealistic or inappropriate assumptions (e.g., genetic equilibrium conditions, narrow ranges of demographic conditions), resulting in biased or imprecise estimates (Faubet et al. 2007, Meirmans 2014, Younger et al. 2017). Also, genetic estimates of demographic connectivity can be highly uncertain (Samarasin et al. 2017). Thus, the practical benefit of using estimates of connectivity based on real-world data to inform optimal allocation of control resources remains poorly understood.

Applying optimal resource allocation strategies through connectivity-based management requires monitoring data on abundance, demographic connectivity, and the damage function. In addition to structural uncertainty in the ecological and management processes that determine the true levels of abundance, the data used by managers to implement connectivity-based management are uncertain due to observational error. Observational uncertainty in monitoring data of invader presence or abundance can make theoretically optimal strategies less effective (Chades et al. 2011, Regan et al. 2011, Rout et al. 2014, Kling et al. 2017, Bonneau et al. 2019). Similarly, as the optimal resource allocation strategy depends on the relationship between invader abundance and damage costs (Yokomizo et al. 2009, Davis et al. 2018a), imperfect knowledge in this relationship could affect the optimal strategy. And, yet to be determined, are the potential effects of imperfect knowledge about the true connectivity structure.

In this study, we concurrently consider the effects of uncertainty in abundance, connectivity, and damage functions in a single framework. Our work builds on studies that have separately examined how observational uncertainty in abundance (Haight and Polasky 2010, Kling et al. 2017) and knowledge of the damage function (Yokomizo et al. 2009, Davis et al. 2018a) affects the optimal resource allocation strategy. Specifically, our objective was to provide guidance for using one-time connectivity estimates to inform surveillance and control decisions in practical settings. Our hypothesis was that observational uncertainty in the one-time estimate of connectivity would decrease the benefits of prioritizing resources spatially because there would be increased error in identifying source populations (i.e., local populations that provide migrants) and their migration rates. We also expected that the optimal resource allocation strategy would depend on knowledge of the damage function and accuracy of the regular abundance estimates that are used to guide spatial prioritization. Our results provide general guidance for spatial prioritization of resources under multiple sources of uncertainty and fixed budgets and highlight which data may be more valuable to collect for optimizing resource allocation.

\section{Methods}

\section{Invasive species and management context}

Our theoretical framework is inspired by demographic dynamics of invasive wild pigs (Sus scrofa) in areas of the United States where populations of wild pigs are not contiguous; although our approach was developed to apply more generally to the control of any spatially 
structured pest population. In areas of the United States where wild pig populations are less abundant and along the edges of areas with high-density pig populations, their distribution is similar to a metapopulation with distinct subpopulations and genetic evidence of recent dispersal between subpopulations (T. Smyser, unpublished data). Wild pigs produce offspring throughout the year (Mayer and Brisbin 2009), with one or two seasonal peaks in parturition rather than one distinct seasonal birth pulse. A genetic archive of wild pig samples collected ancillary to ongoing control efforts is currently being developed to guide management decisions based on spatial dispersal probabilities and directions. We used a range of probabilities and simplified connectivity structures that have been determined for wild pigs from genetic analyses (Tabak et al. 2017, Hernandez et al. 2018; T. Smyser, unpublished data) to determine optimal allocation of resources across populations where the management objective is to eliminate the metapopulation at the lowest overall cost of damages and removal costs.

\section{Overview of approach}

Similar to previous work (Chades et al. 2011, Baker and Bode 2016, Baker 2017), our framework accounts for nonlinear relationships between population density and removal and damage costs, in determining the optimal resource allocation strategy. However, we simplify the number of potential resource allocation strategies a priori by considering practical rules of thumb ("prioritization schemes") that could be implemented by managers at coarse time scales. Thus, our method aims to identify the best strategy for the objective function (defined in Evaluation section) within a pre-defined set of strategies (hereafter referred to as "optimal"), rather than the optimal solution from all possible strategies (i.e., we conducted simulation optimization Amaran et al. 2014, Punt et al. 2016). We define the optimal resource allocation strategy as the one with the highest return on investment (ROI; "returns", i.e., savings from management, divided by its management costs;see Eq. 14).

We use a stochastic metapopulation model to allow for natural levels of uncertainty (stochasticity) that would be expected in the true management (number of individuals removed relative to the intended number) and ecological (abundance in each subpopulation) processes. These sources of uncertainty are structural, affecting the generation of the true abundance. To this, we add observational uncertainty in abundance and connectivity. We assume that the manager has an estimate of connectivity among local populations through a genetic analysis (or other methods) conducted at the outset of a new management program. We also assume that the manager has monitoring data on population abundance at each time step through a method that requires no additional cost, e.g., analyzing the removal data using a removal model (Davis et al. 2016). We first examine how applying different prioritization schemes to different connectivity structures and damage functions affects ROI when abundance and connectivity are perfectly observed. Then, we examine how observational uncertainty in the one-time estimate of connectivity and the monthly estimates of abundance affect ROI of the assumed optimal strategy. Our approach is conceptually similar to bioeconomic models such as Haight and Polasky (2010) and Regan et al. (2011) in that we examine the effects of observational uncertainty on optimal resource allocation strategies. However, our approach differs from these approaches in two important ways: (1) we assume that the manager pre-determines how resources should be allotted in space based on knowledge of connectivity structure and test how observational uncertainty in connectivity and abundance monitoring affects ROI of an assumed optimal resource allocation strategy, rather than determining how much should be apportioned to monitoring throughout control in order to apply the optimal strategy, and (2) our "strategies" are defined by how a fixed amount of resources are apportioned in space rather than how they are apportioned to control vs. monitoring. In our approach, we assume all monitoring data are available at no additional cost.

Code for the simulation model is given in Data S1. The model processes at each time step progress as follows: (1) density-dependent growth of each subpopulation (Eqs. 1, 2), (2) dispersal among subpopulations according to their connectivity structure and rates (Eqs. 3, 4), (3) management removal of individuals from each subpopulation (Eq. 5) dependent on the abundance-capture-success relationship (Eq. 6) and the effort allotted to each subpopulation, which depends on the overall effort available $(\beta)$ and an effort prioritization function $\left(f_{\mathrm{p}}\right)$ among subpopulations (Eqs. 7, 8), (4) determination of management costs dependent on multiplying overall effort by a dollar value (Eq. 9), and, finally, (5) determination of the damage value dependent on density (Eq. 10) and on the shape of the density-damage relationship that is fixed throughout each simulation (either Eqs. 11, 12, or 13). Last, ROI is calculated for each simulation from the cumulative management costs, cumulative damage costs, and elimination status at the end (Eq. 14). Each of these processes is described in detail in the following.

\section{Metapopulation model}

We consider an established metapopulation of an invasive species that is spatially structured with three subpopulations connected through dispersal, and closed to emigration and immigration to and from outside sources. We also assume that each subpopulation has a fixed carrying capacity (i.e., pre-defined geographic capacity) such that subpopulations cannot expand outside their patches. We examine six different connectivity "motifs" within the metapopulation to study the effects of connectivity structure on the optimal management strategy (Fig. 1). We assume that each subpopulation 


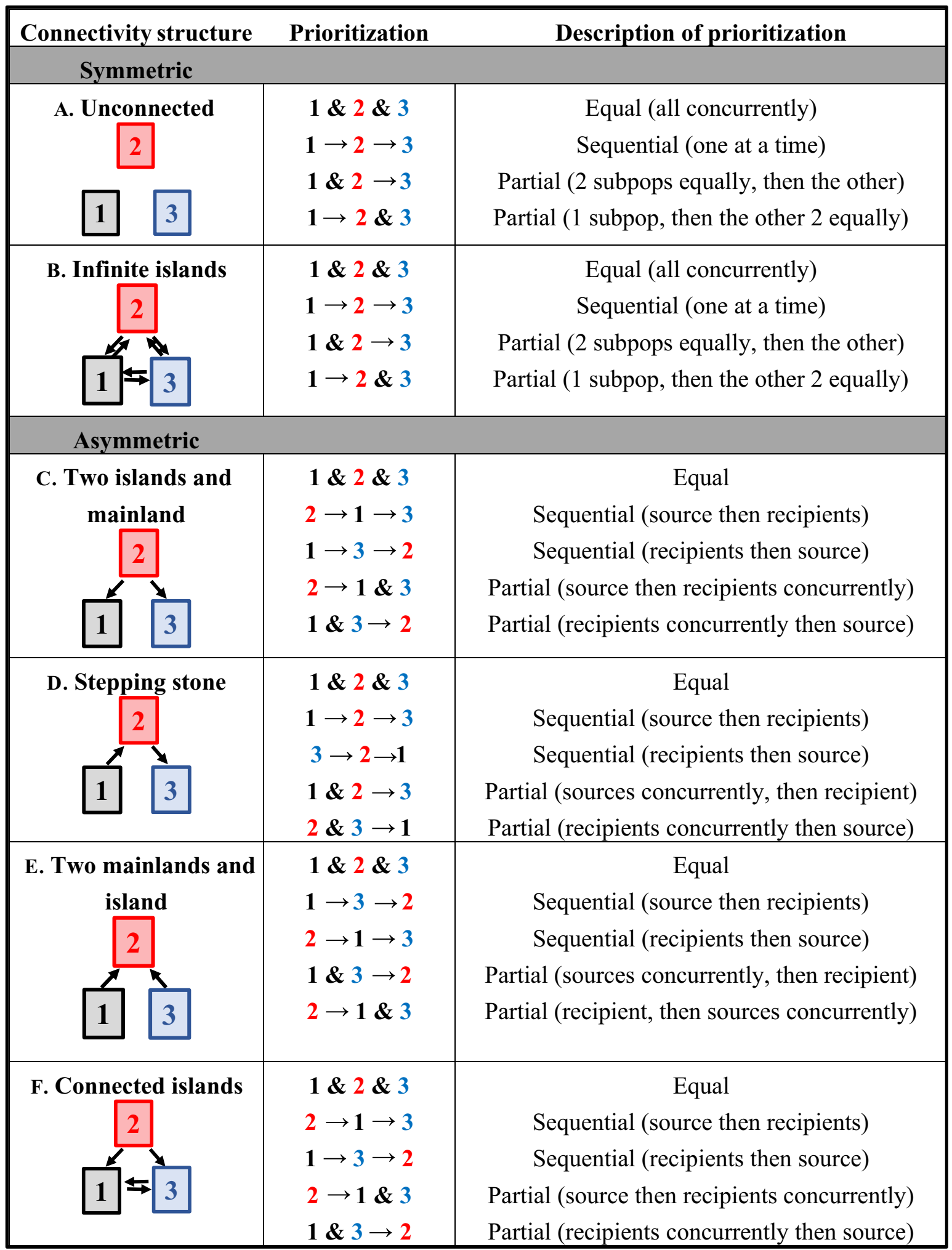

FIG. 1. Connectivity motifs and a description of prioritization schemes. Connectivity increases with the number of arrows. 
can expand locally (up to their carrying capacity) and that subpopulations can be completely removed, but that no new subpopulations can be added. At the start of control, we assume that the metapopulation is at carrying capacity (i.e., equal abundance of subpopulations) and that control efforts decrease metapopulation abundance below carrying capacity.

Reproduction.-We assume subpopulations in the metapopulation undergo logistic population growth to a fixed carrying capacity. Birth dynamics occur in discrete time at a monthly time step with Poisson-distributed error

$B_{t, k} \sim \operatorname{Poisson}\left(\lambda_{t, k}\right)$, where $\lambda_{t, k}=r_{t} N_{t-1, k}\left(1-N_{t-1, k} / K\right)$

with abundance dynamics

$$
N_{t+1, k}=N_{t, k}+B_{t, k}+M_{t, J \rightarrow k}-M_{t, k \rightarrow J}-R_{t, k}
$$

here, $B_{t, k}$ are the new births in month $t$ in subpopulation $k$, and $N_{t, k}$ is population abundance. $M_{j \rightarrow k}, M_{k \rightarrow j}$, and $R_{t, k}$ (described in detail in Dispersal) are the number of immigrants from each subpopulation $j$ to subpopulation $k$, the number of emigrants from subpopulation $k$ to each subpopulation $j$, and the number of invaders removed due to control. We assume the fixed carrying capacity, $K$, is equal to 1,000 in each subpopulation and that abundance can exceed this level slightly due to stochastic processes. The parameter $r_{t}$ is a monthly growth rate that fluctuates based on seasonal birth dynamics (Table 1). Each subpopulation is initialized at carrying capacity.

Dispersal.-Subpopulations are potentially connected by dispersal. We consider six different dispersal structures: (A) unconnected, (B) infinite islands, (C) two islands and mainland, (D) stepping stone, (E) two mainlands and island, and (F) connected islands (Fig. 1). At each time step, new migrants are added to subpopulation $k$ as follows

$$
\begin{aligned}
M_{t, J \rightarrow k}= & \sum_{j=1}^{J} \operatorname{Binomial}\left(N_{t, j}, X_{j \rightarrow k}\right), \text { with constraints } j \\
& \neq k \text { and } M_{t, j \rightarrow k} \leq N_{t, j} \\
M_{t, k \rightarrow J}= & \sum_{j=1}^{J} \operatorname{Binomial}\left(N_{t, j}, X_{k \rightarrow j}\right), \text { with constraints } j \\
& \neq k \text { and } M_{t, k \rightarrow J} \leq N_{t, k}
\end{aligned}
$$

$X_{j \rightarrow k}$ is the monthly dispersal probability from subpopulation $j$ to $k$. Thus, we assume that dispersal can occur in any month, depending on fixed dispersal probabilities among subpopulations, and that dispersal probabilities are proportional to abundance (i.e., not density dependent).
Control. - No prioritization. - In the absence of prioritization, a fixed monthly control effort $(\beta)$ is always divided equally among subpopulations. We assume that the number of individuals removed from a population $k$ in month $t, R_{t, k}$, is a random variable that depends on effort allocated, and declines steeply with the population size as individuals become hard to find (Choquenot et al. 1999). We model $R$ as a Poisson random variable

$$
\begin{aligned}
R_{t, k} \sim & \text { Poisson }\left(N_{t, k} \times f_{\mathrm{a}}\left(N_{t, k}\right) \times \beta / \sum k\right) \\
& \text { with constraint } R_{t, k} \leq N_{t, j}
\end{aligned}
$$

where $f_{\mathrm{a}}$ is the abundance-capture-success function and $\sum k$ is the total number of subpopulations. The $f_{\mathrm{a}}$ function quantifies the success probability of removal efforts based on current abundance. We follow the intuition of (Choquenot et al. 1999), defining $f_{\mathrm{a}}$

$$
f_{\mathrm{a}}\left(N_{t, k}\right)=1-\frac{1}{\left(1+\alpha_{\mathrm{c}}\right)^{N_{t, k}}},
$$

The parameter $f_{\mathrm{a}}$ assumes that the proportion of the population that can be removed using a fixed amount of effort increases sharply at lower abundances to an asymptote of 1 when abundance is high because individuals are more easily located at high abundance and thus removal success (the proportion of the target removal number) increases to full capacity (i.e., 1). We assume that monthly removal success is based on the monthly starting abundance and does not change within a month. For example, if $\alpha_{c}=0.03$ and abundance is $>200$, then the target removal number will almost always be achieved. However, the actual number removed will be lower than the target removal number when abundance is $<200$ pigs with $\alpha_{c}=0.03 . \alpha_{c}$ is a tuning parameter affecting the threshold abundance where actual management removal becomes lower than the target number (Appendix S1: Fig. S1). In general, lower values of $\alpha_{c}$ lead to less efficient removal (lower success), whereas higher values lead to more efficient removal (higher success). By modeling the effects of removal using a Poisson variable (Eq. 5), we allow for stochastic variation in abundance to scale to mean abundance.

Prioritization.-When prioritization of subpopulations occurs, the fixed monthly control effort $(\beta)$ is not divided equally, rather it is apportioned to subpopulations using a prioritization function $\left(f_{\mathrm{p}}\right)$. We assume that the manager has abundance estimates at each time step to determine the amount of effort that should be allotted to each subpopulation based on $f_{\mathrm{p}}$, and that effort would begin to be shifted from the most prioritized subpopulation to less prioritized subpopulations as abundance decreases because fewer locations within a subpopulation would need to be targeted at lower abundance. Thus, in general, when prioritization occurs, the number of individuals removed at time $t$ in subpopulation $k$ is 
TABle 1. Description of parameters.

\begin{tabular}{|c|c|c|}
\hline Parameter & Description & Values \\
\hline \multicolumn{3}{|c|}{ Fixed values } \\
\hline K & $\begin{array}{l}\text { carrying capacity of } \\
\text { each subpopulation }\end{array}$ & 1,000 \\
\hline$r$ & $\begin{array}{l}\text { monthly population } \\
\text { growth rate (January- } \\
\text { December); values } \\
\text { chosen to mimic birth } \\
\text { seasonality in feral } \\
\text { swine-similar to } \\
\text { (Pepin et al. 2017) }\end{array}$ & $\begin{array}{c}0.1069,0.0855,0.0362 \\
0.0378,0.0526,0.0296 \\
0.0247,0.0247,0.0066 \\
0.0247,0.0132,0.0576\end{array}$ \\
\hline$D_{\max }$ & $\begin{array}{l}\text { total cost of damage } \\
\text { over } 10 \text { yr without } \\
\text { control }\end{array}$ & $\$ 720,000$ \\
\hline$P_{\mathrm{r}}$ & $\begin{array}{l}\text { monthly maximum } \\
\text { funding for resources } \\
\text { (effort) available per } \\
\text { subpopulation }\end{array}$ & $\$ 2,000$ \\
\hline$P_{\mathrm{d}}$ & $\begin{array}{l}\text { monthly maximum cost } \\
\text { of damage per } \\
\text { subpopulation }\end{array}$ & $\$ 2,000$ \\
\hline$\alpha_{c}$ & $\begin{array}{l}\text { scaling parameter for } \\
\text { abundance-capture- } \\
\text { success function }\end{array}$ & $0.013,0.03,0.07$ \\
\hline$\alpha_{\mathrm{p}}$ & $\begin{array}{l}\text { scaling parameter for } \\
\text { effort prioritization } \\
\text { functions }\end{array}$ & $\begin{array}{l}0.0025,0.005,0.01, \\
0.03\end{array}$ \\
\hline \multicolumn{3}{|c|}{ Variable inputs } \\
\hline$\beta$ & control effort & $0.2,0.4,0.6,0.8$ \\
\hline$X$ & connectivity structure & structures A-F (Fig. 1) \\
\hline$X_{j \rightarrow k}$ & $\begin{array}{l}\text { annual proportion } \\
\text { dispersing from } \\
\text { subpopulation } j \text { to } k \\
\text { (Tabak et al. } 2017 \text {, } \\
\text { Hernandez et al. 2018) }\end{array}$ & $\begin{array}{c}0.01,0.025,0.05,0.075 \\
0.1,0.125,0.15,0.2\end{array}$ \\
\hline $\begin{array}{l}k_{x} \text { in } \\
f_{\mathrm{p}}(N(t) \\
\left.\left.k_{x}\right)\right)\end{array}$ & prioritization schemes & see Fig. 1 \\
\hline$f_{\mathrm{d}}$ & damage function & $\begin{array}{l}\text { saturating, linear, or } \\
\text { exponential }\end{array}$ \\
\hline$U_{\mathrm{a}}$ & $\begin{array}{l}\text { uncertainty in } \\
\text { abundance; truncated } \\
\text { normal distribution } \\
\text { between } 0 \text { and } 1\end{array}$ & $\begin{array}{l}\text { mean, } \sigma^{2}: 1,0 \text { (none); } \\
0.9,0.1 \text { (low); } 0.9,0.25 \\
\text { (high) }\end{array}$ \\
\hline$U_{X j \rightarrow k}$ & $\begin{array}{l}\text { uncertainty in dispersal } \\
\text { probability }\end{array}$ & $\sim$ uniform $(0,0.2)$ \\
\hline$U_{X}$ & $\begin{array}{l}\text { uncertainty in } \\
\text { connectivity structure }\end{array}$ & $\begin{array}{l}\text { random } 3 \times 3 \text { matrix } \\
\text { with values } \sim \text { uniform } \\
(0,1) \text { rescaled so rows } \\
\text { sum to } 1\end{array}$ \\
\hline
\end{tabular}

$$
\begin{aligned}
R_{t, k} \sim & \text { Poisson }\left(N_{t, k} \times f_{\mathrm{a}}\left(N_{t, k}\right) \times f_{\mathrm{p}}\left(N_{t, k_{x}}\right) \times \beta\right) \\
& \text { with constraint } R_{t, k} \leq N_{t, j}
\end{aligned}
$$

where $f_{\mathrm{p}}\left(N_{t, k x}\right)$ is conditional on the spatial prioritization scheme $\left(k_{x}\right.$, Fig. 1) for removal and current $N_{t, k}$ and $\alpha_{\mathrm{p}}$ according to

$$
f_{\mathrm{p}}\left(N_{t, k_{x}}\right)=1-\frac{1}{\left(1+\alpha_{p}\right)^{N_{t, k_{x}}}}
$$

where $\alpha_{p}$ scales the amount of effort that will be allotted to subpopulation $k_{x}$ (specifies the prioritization scheme) as a function of its abundance. Lower values of $\alpha_{p}$ generate prioritization rules that are closer to equal allocation whereas higher values lead to higher discrepancy in the amount of effort allotted to each subpopulation (Appendix S1: Fig. S2). Appendix S1: Fig. S2 shows the effects of $\alpha_{p}$ on example patterns of effort allotment to the most prioritized subpopulation $\left(k_{x=1}\right)$, followed by secondarily and lastly prioritized subpopulations. In addition to equally dividing resources (as above), we allow three potential prioritization schemes $\left(k_{x}\right)$ : (1) sequential $\left(k_{x=1}\right.$ then 2 then 3$)$, (2) first two equally and then the last $\left(k_{x=1} \& 2\right.$ equally, then 3$)$, and (3) first one and then the other two equally $\left(k_{x=1}\right.$ then $2 \& 3$ equally; Fig. 1$)$. Specific equations defining how effort is allotted under different general prioritization schemes (1-3 above) and values of $\alpha_{\mathrm{p}}$ are described in Data $\mathrm{S} 1$ : culling3.

\section{Removal costs and damage function}

We assume that there is a fixed monthly budget available to control the metapopulation with upper maximum $P_{r}$. When effort is divided evenly among the subpopulations, the maximum monthly effort at high abundance in each subpopulation results in removal of $33.3 \%$ of each subpopulation monthly (i.e., if $\beta=1$, which we do not examine). We use an arbitrary maximum monthly budget of $\$ 6,000$ for control of the metapopulation $(\$ 2,000$ per subpopulation when effort is divided equally; with all monetary amounts expressed in real dollars). Different levels of control effort $(\beta=0.8,0.6,0.4,0.2)$ correspond to decreases in the monthly budget for control by $20 \%$, $40 \%, 60 \%$, or $80 \%$, which translates to maximum monthly removal rates of $26.7 \%, 20.0 \%, 13.3 \%$, and $6.7 \%$, in each subpopulation when abundance is high and resources are divided equally. For example, under the lowest control effort ( $\beta=0.2$ ), the maximum amount of monthly funding available to control is $\$ 1,200$ while the maximum amount of monthly damage at carrying capacity is $\$ 6,000$. Thus, $\beta$ determines both the proportion of individuals that can be removed monthly and the relative difference between damage and control costs. Also, as mentioned above, the same amount of resources becomes less efficient as population density declines such that removal success decreases with the same amount of effort once subpopulations reach low densities. Total monthly amount spent on control is as follows:

$$
C_{t, k_{x}}=f_{\mathrm{p}}\left(N_{t, k_{x}}\right) \times \beta \times P_{\mathrm{r}}, \text { or } C_{t}=\beta \times P_{\mathrm{r}}
$$

where $C_{t, k x}$ is the amount spent on subpopulation $k_{x}$ in month $t, C_{t}$ is the amount spent for all subpopulations in month $t$, and $P_{r}$ is the fixed maximum amount of resources available to be spent each month for removal $(\$ 6,000)$.

We examine three different damage functions $\left(f_{\mathrm{d}}\left(N_{t}\right.\right.$, $k$ )) for determining damage costs based on abundance (Eqs. 11-13). We assume that total monthly damage 
costs from the metapopulation $\left(D_{t}\right)$ at carrying capacity are a fixed arbitrary value $(\$ 6,000$ for the metapopulation; $\$ 2,000$ per subpopulation)

$$
D_{t}=\sum_{k=1}^{k=3} f_{\mathrm{d}}\left(N_{t, k}\right) \times P_{\mathrm{d}}
$$

where $P_{\mathrm{d}}$ is the maximum cost of damage for a subpopulation when it is at carrying capacity $(\$ 2,000)$ and $f_{\mathrm{d}}$ is the proportional damage as a function of current abundance. The different damage functions are described as follows:

$$
\begin{gathered}
f_{\mathrm{d}}=\left(1-\frac{1}{(1+100)^{\frac{N_{t, k}^{K}}{K}}}\right) \quad \text { (saturating) } \\
f_{\mathrm{d}=\frac{N_{t, k}}{K}} \quad \text { (linear) } \\
f_{\mathrm{d}}=\frac{1}{(1+100)^{1-\frac{N_{t, k}}{K}}} \quad \text { (exponential). }
\end{gathered}
$$

These damage functions are similar to Type II, Type I, and Type IV functional responses (Holling 1959), except our Type IV (exponential) function does not saturate at high abundance after rising from the low damage condition (Appendix S1: Fig. S3). We assumed the damage function was fixed throughout each simulation. We tested the effects of imperfect knowledge of the damage function by determining the optimal resource allocation strategy for each damage function.

\section{Evaluation}

We evaluated outcomes of simulations by ROI, a measure of cost effectiveness. ROI calculates the difference between total costs in the absence of control and total costs with control (i.e., damages avoided net of control costs) scaled to the cost of control. Thus, ROI gives the amount saved per dollar spent on control. ROI was calculated as

$$
\mathrm{ROI}=\frac{D_{\max }-\left(\sum C_{t}+\sum D_{t}\right)}{\sum C_{t}} \times \mathbb{I}_{e} \times 100 \%
$$

where $D_{\max }$ is the total damage cost over $10 \mathrm{yr}$ when no control occurred, and $\mathbb{I}_{e}$ indicates whether the metapopulation was eliminated $\left(\mathbb{I}_{e}=1\right)$ or not $\left(\mathbb{I}_{e}=0\right)$ by the end of the simulation. Thus, we assumed that runs that did not eliminate had a return on investment of 0 because our objective was to identify strategies that led to elimination with the highest return on investment.

\section{Observational uncertainty in abundance}

Our prioritization methods assumed that the manager has monitoring data that can be used to estimate abundance in the subpopulations at each time step (Davis et al. 2016). Because abundance measures are typically imperfect, we examined how uncertainty in the monthly estimates impacts ROI of the optimal prioritization plan (Kendall and Moore 2012). We examined two levels of uncertainty in abundance $\left(U_{\mathrm{a}}\right.$ : low and high). We assumed that the process for observing abundance is biased low (underestimation of the true abundance) because studies that have estimated detection probability during management-based monitoring in populations have found that it is well below 1 (Davis et al. 2018b, although in the supplementary information we also present results for instances where abundance estimation is imperfect but unbiased; Appendix S1: Fig. S10). We assumed the error term is a normally distributed random variable truncated between 0 and 1 (i.e., biased low), with mean 0.9 and variance of 0.1 (low error) or 0.25 (high error). For each subpopulation at each time step, we multiplied the random proportion variable by the true abundance to determine the "observed" abundance. Then, effort prioritization at each month was determined based only on the observed abundance and the prioritization function $\left(k_{x}\right.$ scheme and $\left.\alpha_{\mathrm{p}}\right)$.

\section{Observational uncertainty in connectivity}

Because measures of connectivity can be difficult to obtain, we assumed that the manager had connectivity information once at the start of control (one-time information). We examined the situation where the manager assumes the one-time connectivity information is perfectly observed and remains the same over time, and thus applies the optimal control strategy in the case where abundance and connectivity are observed perfectly. To evaluate effects of uncertainty in connectivity structure, we apply the optimal strategy for each connectivity structure to itself and every other connectivity structure and calculate the mean and variance in ROI across all connectivity structures. Thus, our methodology evaluates how observing the wrong connectivity structure and thus potentially applying a suboptimal allocation strategy affects the ROI outcome. Note that for some connectivity structures the same allocation strategy is optimal and thus observing connectivity structure imperfectly may not be problematic. We tested both aspects of imperfect knowledge in connectivity: the structure (pathways in Fig. 1) and the rates (dispersal probability among the edges). To evaluate effects of uncertainty in structure, we held the rate constant at 0.1 and evaluated ROI across all six structures under each optimal control strategy. To evaluate the effects of rates, we held the structure constant and evaluated ROI across all dispersal probabilities (Table 1). When evaluating uncertainty in both components we evaluated ROI for all structures and dispersal probabilities. In order to compare the uncertainties on equal grounds, we calculated the means and their coefficients of variation by subsampling the 
data at random so that each metric was composed of 100 data points because there were more data points when both types of uncertainties were combined.

\section{Simulations}

Each subpopulation was initialized with 1,000 individuals and thus an equal level of damage. Each parameter set was run for $10 \mathrm{yr}$ of population dynamics and replicated 100 times. Design of parameter sets in simulations is given under "variable inputs" in Table 1. To evaluate the effects of observational uncertainty on the optimal resource allocation strategy, we only ran the optimal spatial prioritization schemes (each optimal scheme ran on all six connectivity structures), and fixed $f_{\mathrm{d}}$ at linear, $\alpha_{p}$ at 0.03 , and $\alpha_{c}$ at 0.03 , because effects of these parameters were examined in the first set of analyses (without uncertainty in abundance and connectivity). We did include variation in $\beta$ in the uncertainty analyses because it was such a strong predictor of ROI. We performed all simulations and analyses in Matlab R2018a (MathWorks, Natick, Massachusetts, USA). We evaluated how observational uncertainty in abundance and connectivity affected the optimal resource allocation strategies using (1) descriptive summaries of mean ROI and its uncertainty in terms of a coefficient of variation (reported below), (2) random forest analysis on the ROI outcomes to examine the relative importance of variables (methods described in caption of Appendix S1: Fig. S8), and (3) a zero-inflated negative binomial model on the ROI response to estimate the direction of effects of different variables (methods described in caption of Appendix S1: Table S1).

\section{RESULTS}

\section{Control effort and damage function}

Under the lowest control effort $(\beta)$ and no connectivity or prioritization $(0.2$; removal of $6.7 \%$ of the metapopulation monthly at carrying capacity; budget of $\$ 1,200$ monthly), control efforts failed to eliminate the metapopulation within $10 \mathrm{yr}$, even when the abundancecapture-success parameter $\left(\alpha_{c}\right)$ was high (Appendix S1: Fig. S4), unless the subpopulations were sequentially prioritized (Appendix S1: Fig. S4 vs. S5). At a removal intensity of $0.4(13.3 \%$ of the metapopulation monthly at carrying capacity; budget of $\$ 2,400$ monthly), when there was no prioritization complete elimination only occurred sometimes unless $\alpha_{c}$ was high (Appendix S1: Fig. S4). However, if the subpopulations were sequentially prioritized, elimination occurred more often (ROI was higher) even when $\alpha_{c}$ was moderate (Appendix S1: Fig. S5). A control effort of at least $0.6(20 \%$ of the metapopulation monthly at carrying capacity; budget of $\$ 3,600$ monthly) and a moderate or high $\alpha_{c}(0.03,0.07)$ was required to eliminate the metapopulation frequently, with ROI being higher when removal was sequentially prioritized. (Appendix S1: Figs. S4, S5). Some form of prioritization almost always outperformed equal allocation of effort across the range of control effort values, except when the subpopulations had full, symmetric connectivity (Appendix S1: Fig. S6), but the magnitude of benefit from prioritization depended on control effort (Appendix S1: Fig. S6E). Relative to the effects of $\beta$, the effects of the damage function $\left(f_{\mathrm{d}}\right)$ were more subtle (Fig. 2A vs. B). ROI was generally higher when $\alpha_{\mathrm{p}}$ (scaling parameter of the prioritization function) was higher, meaning that more stringent prioritization (less like equal allocation) was generally better (Fig. 2C). Capture success (controlled by $\alpha_{c}$ ) had a very strong effect on ROI (Fig. 2D), where more efficient capture (higher values of $\alpha_{c}$ ) lead to much higher ROI.

In general, ROI tended to be higher when the damage function was exponential but the magnitude of effect depended on $\beta$ (Appendix S1: Figs. S4, S5) and connectivity structure (Fig. 2B). While absolute ROI was affected by the damage function, prioritization of the source (either sequential or partial) remained optimal across all three damage functions (Fig. 3C-F) for the full range of $\beta$ (data not shown). However, the damage function did affect the optimal strategy when connectivity was symmetric (Fig. 3A, B).

\section{Connectivity and dispersal probability}

For asymmetric connectivity, ROI was always higher when sources were prioritized, regardless of dispersal probability values (Fig. 4). However, when subpopulations had full symmetric connectivity, equal resource allocation was best at high levels of dispersal probability, while unequal resource allocation was better at lower values of dispersal probability (Fig. 4). When there were multiple source populations, it was optimal to prioritize both sources at the same time rather than to allot resources to each sequentially before allotting resources to the recipient (Fig. 4D, E). The best strategies were optimal both in terms of mean and uncertainty in ROI, i.e., in general, strategies that predicted the highest mean ROI predicted the lowest uncertainty in ROI relative to their means (or at least similarly low uncertainty : mean ROI ratios as competing strategies; Appendix S1: Fig. S7), indicating that the best strategies were also appropriate for risk-averse managers.

\section{Uncertainty}

Uncertainty in abundance and connectivity structure led to lower ROI (Fig. 5A-C) with higher uncertainty in ROI (Fig. 5D-F), but the magnitude of effects on mean ROI depended on control effort (Fig. 5; Appendix S1: Fig. S8, Appendix S1: Table S1 rows 34-36). The effects of uncertainty in abundance and connectivity structure tended to be strongest at moderate values of control effort $(\beta=0.4$ or 0.6; Fig. 5, Appendix S1: Fig. S9). When all types of observational uncertainty were present and $\beta=0.6$, ROI 
A

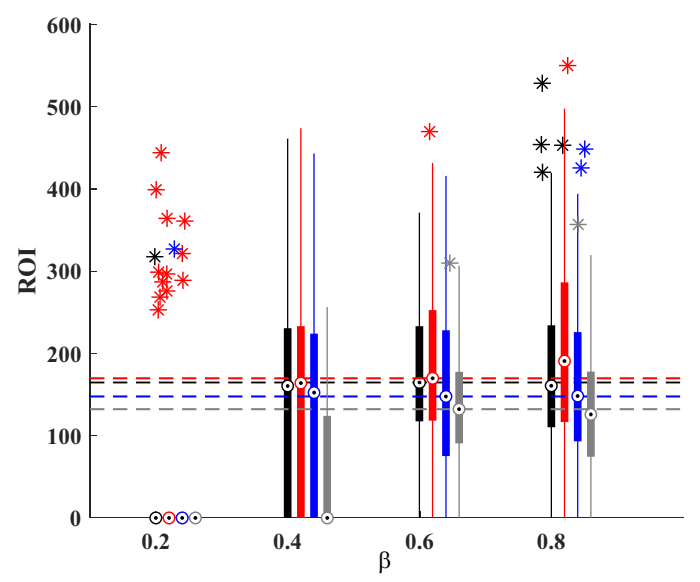

C

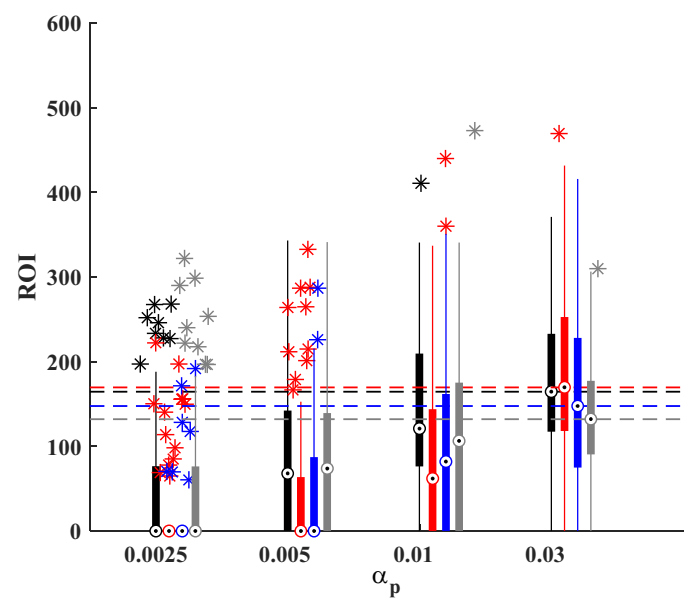

B

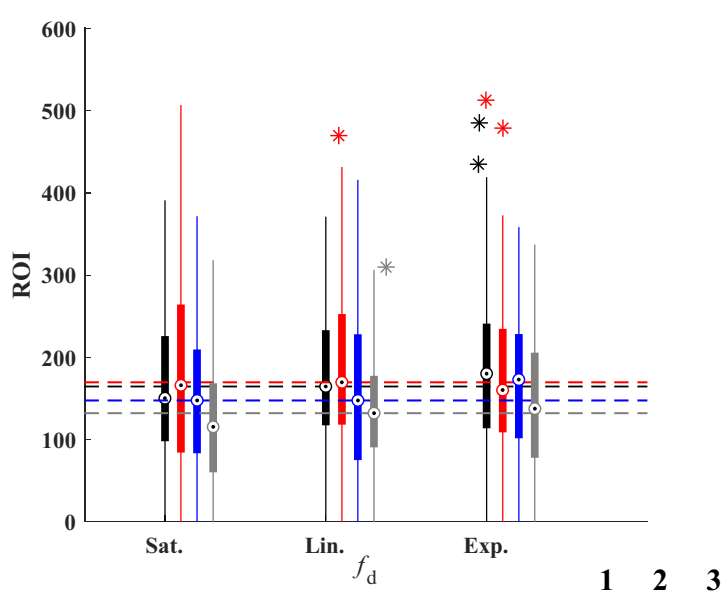

$1 \leftarrow 2 \rightarrow 3$

D

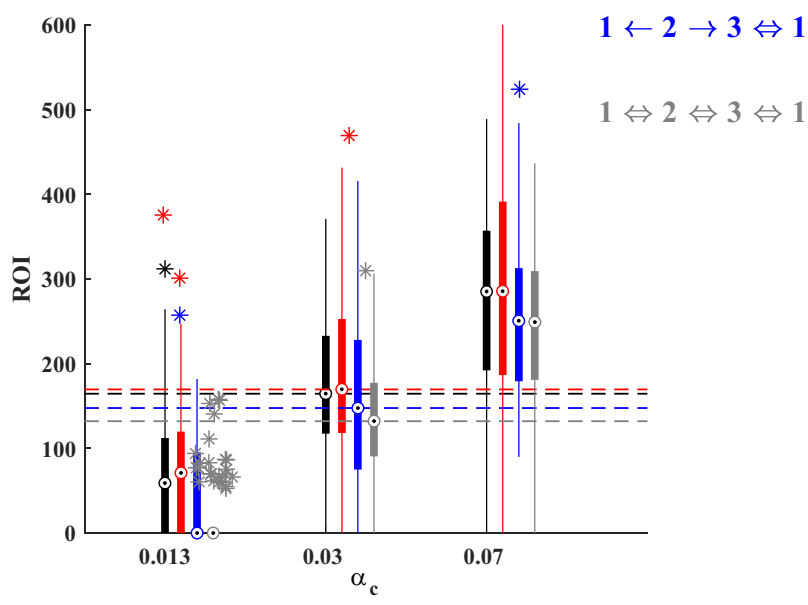

FIG. 2. One-way sensitivity analysis of key parameters on return on investment (ROI, \%) for the optimal prioritization rule (shown as stars in Fig. 3). (A) Effects of control intensity (budget); (B) damage function (saturating, linear, exponential); (C) prioritization parameter, $\alpha_{\mathrm{p}}$; (D) capture success parameter, $\alpha_{\mathrm{c}}$. Box plots summarize ROI for 100 replicate simulations (mid dot shows the median, box edges span the 25th to 75th percentile, whiskers show span the full range of data that are not outliers). Each series of box plots represents results from a different connectivity structure (see legend). Stars are outliers in the box plots distributions. Horizontal dashed lines indicate the median ROI for the default parameter values (i.e., values that were fixed in Fig. 2 ; $\beta=0.6$, $f_{\mathrm{d}}=$ linear, $\alpha_{\mathrm{c}}=0.03$, and $\left.\alpha_{\mathrm{p}}=0.03\right)$.

was $57 \%$ and $73 \%$ lower on average in cases where the true connectivity was none or asymmetric, respectively. Uncertainty in connectivity rate was less important than uncertainty in connectivity structure and abundance (Fig. 5; Appendix S1: Figs. S8, S9). The negative effects of uncertainty in abundance were not significantly different across true connectivity structures (Appendix S1: Table S1 rows 19-23), while the magnitude of effects of uncertainty in connectivity structure and rate on ROI depended significantly on the true connectivity structure (Fig. 5; Appendix S1: Table S1 rows 24-33). There was a significantly negative interaction between uncertainty in connectivity rate and structure (Appendix S1: Table S1 row 18), but no significant interaction between uncertainty in abundance and connectivity structure or rate (Appendix S1: Table S1 rows 16-17).

\section{DisCUSSION}

Ecological and bioeconomic theory suggests that spatial prioritization of resources is important for efficient control of invasive species or disease (Hanski and Ovaskainen 2000, Chades et al. 2011, Hastings 2014, Baker 2017). However, applying spatial prioritization plans in practice requires data that describe the connectivity between subpopulations in space. As quantifying demographic connectivity directly can be time consuming and expensive, one way to infer movement between 
A

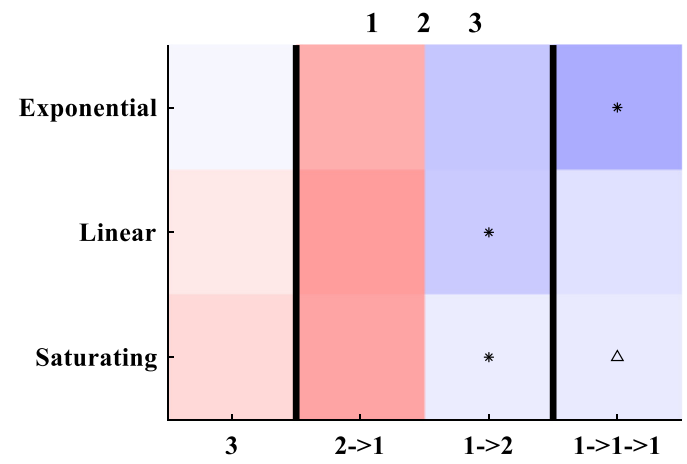

C

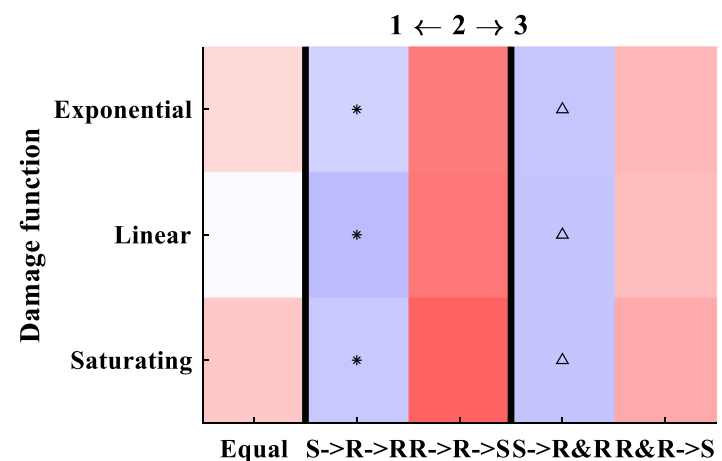

$\mathbf{E}$

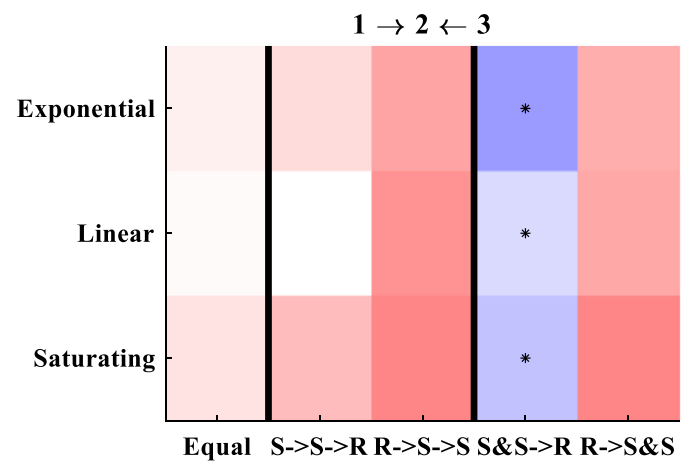

B

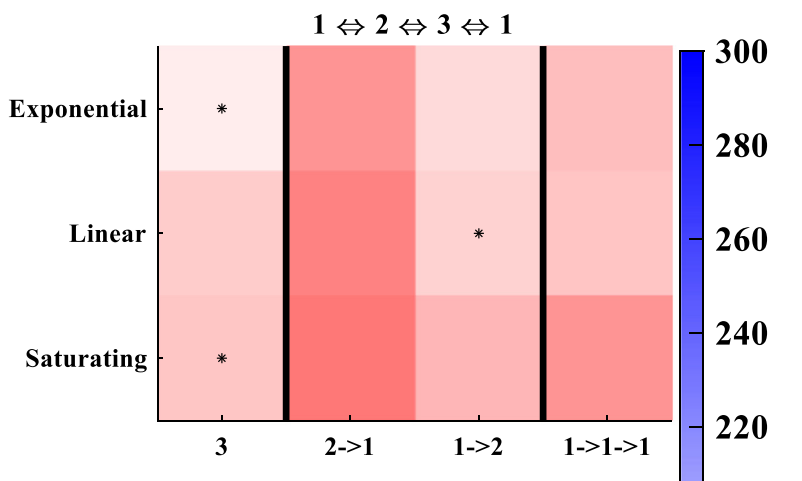

D

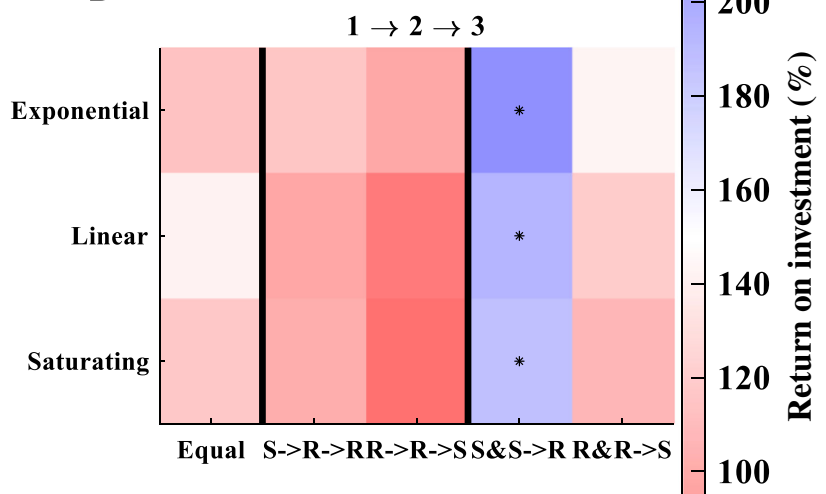

F

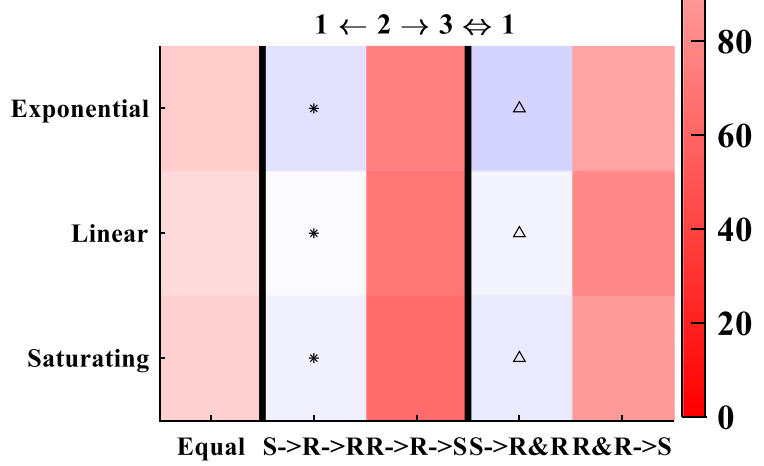

FIG. 3. Effects of damage function on ROI and optimal strategy. Colors represent mean ROI of 100 replicate simulations. Each plot shows results for a different fixed connectivity structure. The $x$-axes for symmetric connectivity structures (A, B) indicate the number of subpopulations that are prioritized simultaneously (e.g., 3 means that all subpopulations are equally prioritized, whereas $2 \rightarrow 1$ means that two subpopulations were initially prioritized simultaneously followed by the third). The $x$-axes of the asymmetric connectivity structures $(\mathrm{C}-\mathrm{F})$ indicate the order of source $(\mathrm{S})$ and recipient $(\mathrm{R})$ prioritization, where "Equal" refers to distributing all resources equally, "\&" indicates that two subpopulations are being prioritized simultaneously, and an arrow indicates sequential prioritization. The $y$-axes indicate the shape of the damage function. Fixed conditions were $\beta=0.6, \alpha_{c}=0.03, \alpha_{p}=0.03$, dispersal probability $=0.1$, abundance uncertainty: none, connectivity uncertainty: none. Stars indicate the "optimal" strategy; triangles are not significantly different from the stars.

subpopulations is to collect genetic samples from the subpopulations and analyze the data using population genetic approaches (Lowe and Allendorf 2010). Here, we showed that uncertainty in directionality of dispersal can substantially reduce ROI and increase its uncertainty. Our results highlight an important challenge for practitioners and geneticists because current methodologies for estimating connectivity using genetics can be highly uncertain or biased unless true demographic processes meet specific conditions (Meirmans 2014, Samarasin et al. 2017), and connectivity structure can be dynamic. The silver lining is that uncertainty in dispersal rates seemed less important than uncertainty in dispersal direction, and genetic methods typically have less uncertainty in estimating dispersal direction than in estimating dispersal rates (Samarasin et al. 2017). 

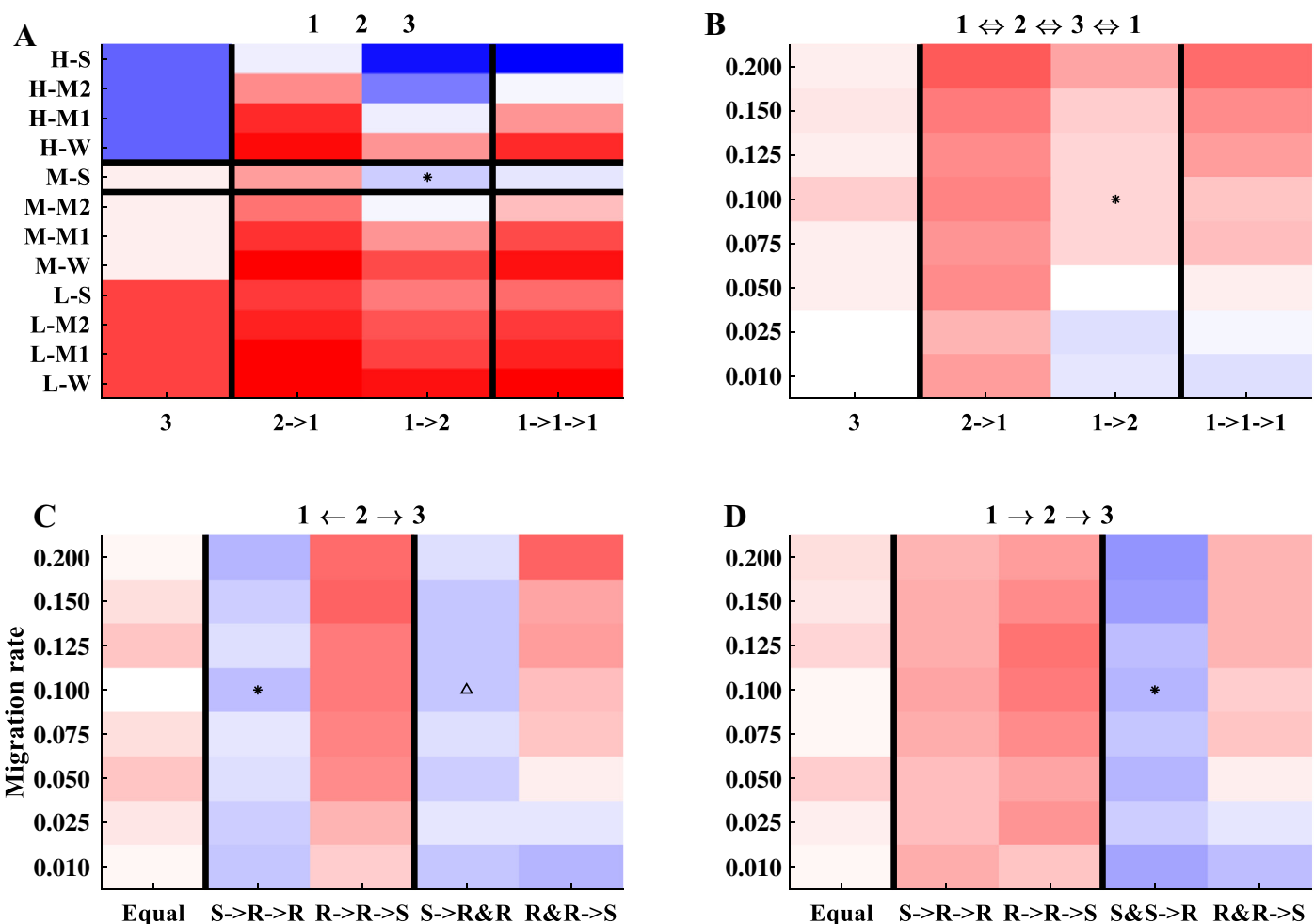

Equal $S->R->R \quad R->R->S \quad S->R \& R \quad R \& R->S$

Equal S->R->R R->R->S S\&S->R R\&R->S

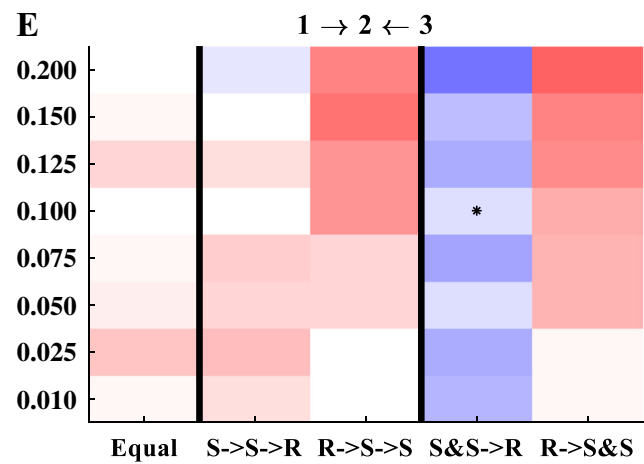

F

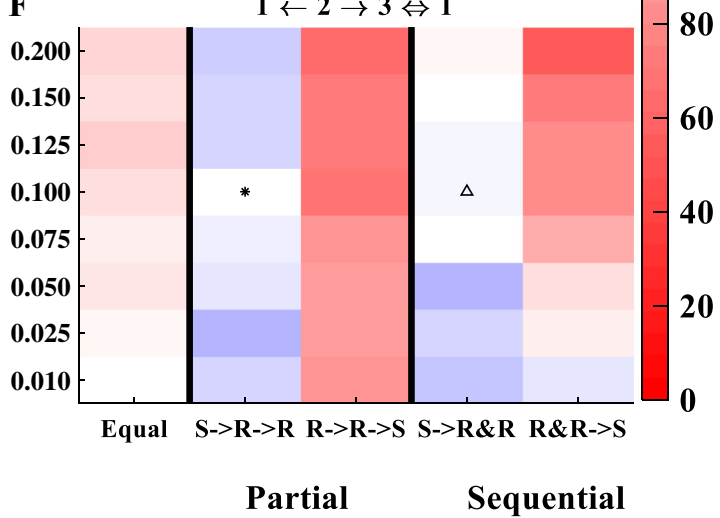

180 胥

\section{Partial Sequential}

Partial Sequential

FIG. 4. Effects of prioritization strategies on ROI (\%). Colors represent mean ROI of 100 replicate simulations. Each plot shows results for a different fixed connectivity structure. The $x$-axes for symmetric connectivity structures (A, B) indicate the number of subpopulations that are prioritized simultaneously (e.g., 3 means that all subpopulations are equally prioritized, whereas $2 \rightarrow 1$ means that two subpopulations were initially prioritized simultaneously followed by the third). The $x$-axes of the asymmetric connectivity structures $(\mathrm{C}-\mathrm{F})$ indicate the order of source/recipient prioritization, where "Equal" refers to distributing all resources equally, "\&" indicates that two subpopulations are being prioritized simultaneously, and an arrow indicates sequential prioritization. The $y$-axes are population-level annual dispersal probabilities (B-F), or levels of the $\alpha_{\mathrm{c}}$ and $\alpha_{\mathrm{p}}$ parameters (A, where H-, M-, L- correspond to high (0.07), medium (0.03), and low (0.013) values of capture success $\left(\alpha_{c}\right)$ as shown in Appendix S1: Fig. S1, and S, M1, M2, and W correspond to strong (0.03), moderate $2(0.01)$, moderate $1(0.005)$, and weak $(0.0025)$ values of prioritization strength $\left(\alpha_{p}\right)$ as shown in Appendix S1: Fig. S2). The horizontal black lines in A show results for the values of $\alpha_{c}=0.03$ and $\alpha_{\mathrm{p}}=0.03$ that are used in plots B-F (note condition A has no potential for dispersal). Other fixed conditions were: $\beta=0.6, f_{\mathrm{d}}=$ linear, abundance uncertainty: none, connectivity uncertainty: none. Stars indicate the "optimal" strategy under these conditions and the average dispersal probability when there is no uncertainty; triangles are not significantly different from the stars.

In practical applications, it is important to understand how budget constraints affect the optimal control strategy in order to use the available resources efficiently
(Epanchin-Niell and Hastings 2010). We found that it was better to prioritize source populations, similar to Baker (2017), across a range of monthly budgets when 
connectivity was asymmetric. However, the optimal resource allocation strategy was affected by budget under no connectivity due to the non-linear relationship between capture success and abundance. In these cases, some form of prioritization was always advantageous but whether the strategy should be partial or sequential depended on budget. Our finding that the budget can affect not just the amount of damage prevented but also the strategy that would lead to the highest ROI and its uncertainty is consistent with previous work based on empirical data from willow (Salix cinerea) invasion management in the Australian Alps (Giljohann et al. 2011). For example, Giljohann et al. (2011) found that the budget affected which sites should be included in the control plan as well as time investment in each site. In general, under low budgets and high connectivity (symmetric or asymmetric), prioritization had little advantage over equal allocation of resources because elimination probability was low for all prioritization schemes under these conditions. Thus, ultimately the optimal resource allocation strategy depends most strongly on whether there are enough resources to rapidly reduce each subpopulation and dispersal probabilities when prioritization is applied (i.e., removal rates that are well beyond birth and immigration rates).

Another important factor determining the optimal resource allocation strategy was the damage function.
Previous work has demonstrated that ROI from control can vary dramatically depending on the damage function and the relative difference of damage costs to control costs (Yokomizo et al. 2009, Davis et al. 2018a). Specifically, when the damage function is saturating and damage costs are low to moderate, there can be little to gain by investing in control (Yokomizo et al. 2009, Davis et al. 2018a). Similarly, we also found that ROI for the optimal strategy was generally lower when the damage function was saturating or linear as compared to exponential. As with the effects of budget, we found that the damage function only affected the optimal resource allocation strategy when connectivity was symmetric, although it did affect absolute ROI under all connectivity structures. Thus, the damage function plays an important role in determining overall ROI, and the optimal strategy depending on the true connectivity structure. Future work should evaluate the ROI of allocating resources into monitoring to decrease uncertainty in the damage function, e.g., see Moore and Runge (2012) for a similar concept.

A third important factor affecting the optimal strategy was observational uncertainty in abundance and connectivity. Our model assumed that the manager allocated resources with a predetermined prioritization scheme based on knowledge of connectivity structure and monthly estimates of subpopulation abundances.

\section{A}

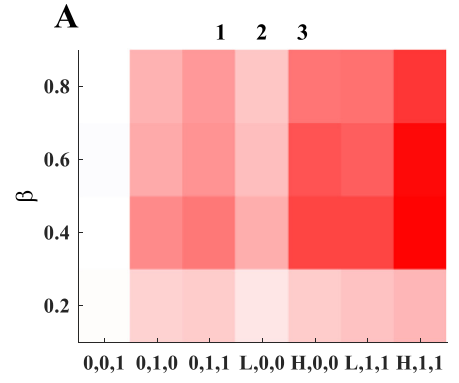

D

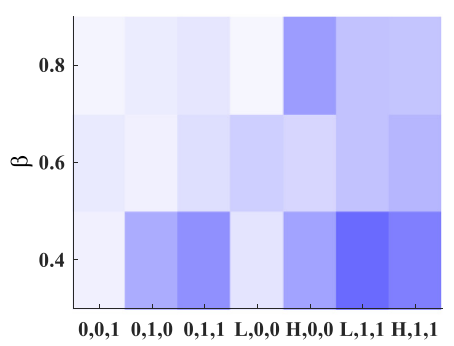

B

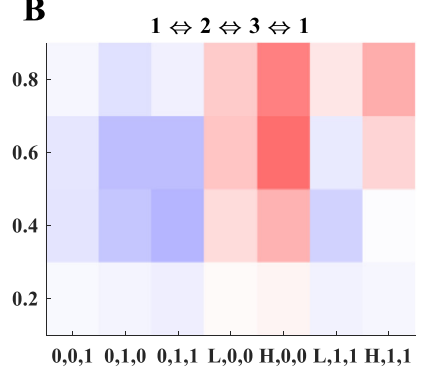

$\mathbf{E}$

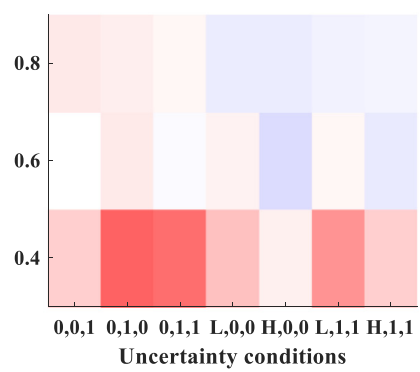

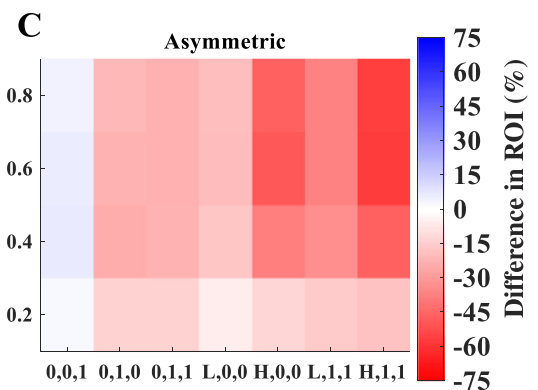

$\mathbf{F}$

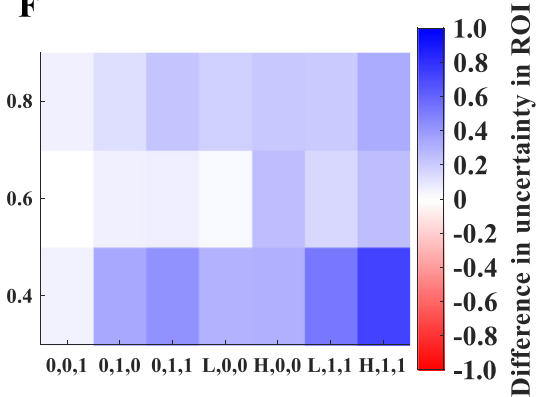

FIG. 5. Effects of uncertainty in abundance and connectivity on ROI (A-C) and its error (D-F). The $x$-axes define the uncertainty conditions $\left(0,0,1=U_{X j \rightarrow k} ; 0,1,0=U_{X} ; 0,1,1=U_{X j \rightarrow k} \& U_{X} ; \mathrm{L}, 0,0=\right.$ low $U_{\mathrm{a}} ; \mathrm{H}, 0,0=$ high $U_{\mathrm{a}} ; \mathrm{L}, 1,1=$ low $U_{\mathrm{a}} \& U_{X j \rightarrow k} \&$ $U_{X} ; \mathrm{H}, 1,1=$ high $U_{\mathrm{a}} \& U_{X j \rightarrow k} \& U_{X}$; see Table 1 for parameter definitions). A-C shows the difference in mean ROI (predictions from model in Appendix S1: Table S1) for each set of uncertainty conditions as compared with no uncertainty, whereas D-F show the difference in coefficient of variation (CV of the raw data) between conditions with uncertainty vs. those without. Note, when $\beta=0.2$, the CVs were generally very high and stochastic even with no uncertainty in connectivity and abundance so these were omitted. True connectivity structures that were evaluated are indicated at the top of the plots; "asymmetric" refers to the average of all four connectivity structures that were not symmetric. 
Thus, when uncertainty in subpopulation abundance was high, subpopulations may have received more or fewer resources than if abundance had been observed more accurately. This allocation error resulted in decreased and more uncertain ROI, similar to Bonneau et al. (2019) and Regan et al. (2011) and occurred regardless of the true connectivity structure. Uncertainty in connectivity did not explain as much of the variation in ROI for different resource allocation strategies as compared to uncertainty in abundance. However, when uncertainty in connectivity structure was present, higher control intensities were needed in order to have more certainty about ROI of the optimal strategy. This was because when the connectivity structure was uncertain, there was error in identifying the true source population (s) such that benefits from reducing dispersal probabilities were reduced. Also, when the true connectivity structure was "completely connected," ROI was higher and less uncertain when there was uncertainty in connectivity structure (data not shown). This was because ROI in several other connectivity structures tended to be higher than ROI in fully connected populations across several different prioritization strategies (i.e., the fully connected case was generally a worst case scenario such that if connectivity was less than fully connected applying any strategy would still give a higher ROI). In contrast, uncertainty in connectivity structure generally decreased expected ROI and increased its uncertainty for all other types of true connectivity structures. Thus, uncertainties from both abundance and demographic connectivity are important for determining optimal resource allocation strategies. Incorporating both sources of demographic uncertainty, in a manner that appropriately links and propagates their uncertainties will be important for determining optimal resource allocation strategies in practice.

While our model accounted for several realistic nonlinear relationships of management costs and density (e.g., similar to Baker 2017), we did not consider density-dependent dispersal between subpopulations, nor dispersal outside the original area occupied by the metapopulation. Instead, we modeled dispersal as a fixed proportion of the source population and without regard to recipient population density. While density-independent dispersal could be unrealistic in some contexts (Olsson et al. 2006, Strevens and Bonsall 2011, Tabak et al. 2017, De Bona and Bruneaux 2019), density-independent connectivity could occur in some situations where anthropogenic movement shapes the connectivity structure (Hernandez et al. 2018). Regardless, we expect that incorporating density-dependent dispersal may have only a minor impact on the relative importance of factors affecting the optimal resource allocation strategy because we found that the details of connectivity structure were less important than the overall control effort and uncertainty in abundance. Although patterns of dispersal were density independent, we assumed that birth rates in local populations were density-dependent. Limiting subpopulations by density-dependent births could affect the optimal prioritization strategy because uncontrolled populations would be allowed to expand further, causing more damage, and resource allocation decisions and damage functions are dependent on densities. Thus exponential growth could offset the benefits of spatial prioritization (depending on spatial expansion rates and damage costs; e.g., Bonneau et al. 2017). Exponential growth in local populations could be realistic in the case of wild pigs in the United States, where uncontrolled populations continue to expand more than controlled populations (Pepin et al. 2019), i.e., they are not limited by favorable habitat. An understanding of density dependence in demographic processes will be important for applying connectivity-based management on real landscapes.

We tested the concept that managers could use a onetime estimate of demographic connectivity that was obtained at the outset of a management program to identify an optimal resource allocation scheme. We then tested how uncertainty in that one-time estimate would affect robustness of the optimal resource allocation strategy that is applied. We assumed that, for a given scenario, connectivity structure and rate were fixed. As connectivity is likely to change due to management or environmental factors, developing bioeconomic frameworks that account for these effects, and how additional surveillance and learning could optimize connectivitybased management plans, will be important for realizing the full benefits of connectivity-based management in practice.

Our results highlighted the importance of accurate monitoring data for planning optimal resource allocation strategies: uncertain knowledge of demographic processes and economic relationships can result in choosing resource allocation strategies with lower ROIs. However, we did not consider the costs of different types of monitoring data (e.g., abundance, connectivity, damage costs). An important future direction is to determine how much investment in monitoring demographic and economic processes would be needed (if any) to maximize ROI by increasing the confidence in choosing the optimal connectivity-based control strategy (EpanchinNiell et al. 2012, Moore and Runge 2012, Rout et al. 2014, Baker and Bode 2016, Gormley et al. 2016). Our results suggest that robust estimates of connectivity will be especially important when connectivity is asymmetric, and that reduced uncertainty in economic data is most important when connectivity is symmetric. Future work that considers the monitoring costs for reducing uncertainties in the data used to guide management decisions is important for determining how resources can optimally be apportioned to control and monitoring in realistic contexts (i.e., where uncertainty in demographic dynamics and damage functions exists).

Our qualitative results should be generally applicable across a wide variety of animal systems where reproduction occurs throughout the year and migration among 
distinct local populations occurs (e.g., rodents, feral ungulates, feral cats). In fact, similar metapopulation modeling approaches have proved useful for informing optimal control in very different types of species, for example, aquatic species such as lionfish (Tamburello et al. 2019) or insects such as gypsy moths (Bogich et al. 2008). Although we did not consider systems with distinct annual birth pulses, we do not expect results to differ for these systems. To understand the importance of uncertainty in dispersal structure, we considered a broad range of connectivity structures, where some will be more relevant than others in specific contexts. For example, the "Stepping-stone" connectivity structure is likely to be most relevant to invasions in streams where dispersal occurs through unidirectional water flow rates. In these systems, our "Infinite islands" structure is not likely to be relevant and thus need not be considered. Thus, some understanding of the dispersal structure in the system being managed is important for identifying the breadth of possibilities that could contribute to uncertainty. Also, in systems where dispersal occurs more continuously across space (e.g., passive dispersal without distinct local populations as in some weed species (Adams et al. 2015) or animal invasions that cover widespread areas of establishment such as wild pigs in Texas), a different model structure for dispersal (i.e., connectivity) would be needed. In these cases, the concept of uncertainty in connectivity structure might be best studied in terms of uncertainty in dispersal rates across a continuous landscape, a process that can also be measured by genetic methods. Our results highlight that regardless of the nature of dispersal structure, its uncertainty is important to consider.

\section{ACKNOWLEDGMENTS}

We thank Gabriel Gellner, David Wolfson, and Michael Tabak for helpful discussion during the development of this work, as well as two anonymous reviewers whose comments greatly improved the manuscript. K. M. Pepin, A. J. Davis, T. J. Smyser, C. Slootmaker, and S. McKee were funded by the United States Department of Agriculture, Animal and Plant Health Inspection Service's National Feral Swine Damage Management Program. Any use of trade, firm, or product names is for descriptive purposes only and does not imply endorsement by the U.S. Government. All authors contributed to conceiving the study and editing the manuscript. K. M. Pepin wrote the code, conducted the analyses, and wrote the paper.

\section{Literature Cited}

Adams, V. M., A. M. Petty, M. M. Douglas, Y. M. Buckley, K. B. Ferdinands, T. Okazaki, D. W. Ko, and S. A. Setterfield. 2015. Distribution, demography and dispersal model of spatial spread of invasive plant populations with limited data. Methods in Ecology and Evolution 6:782-794.

Amaran, S., N. V. Sahinidis, B. Sharda, and S. J. Bury. 2014. Simulation optimization: a review of algorithms and applications. Quarterly Journal of Operations Research 12:301-333.

Baguette, M., S. Blanchet, D. Legrand, V. M. Stevens, and C. Turlure. 2012. Individual dispersal, landscape connectivity and ecological networks. Biological Reviews 88:310-326.
Baker, C. M. 2017. Target the source: optimal spatiotemporal resource allocation for invasive species control. Conservation Letters 10:41-48.

Baker, C. M., and M. Bode. 2016. Placing invasive species management in a spatiotemporal context. Ecological Applications 26:712-725.

Bogich, T. L., A. M. Liebhold, and K. Shea. 2008. To sample or eradicate? A cost minimization model for monitoring and managing an invasive species. Journal of Applied Ecology 45:1134-1142.

Bonneau, M., F. A. Johnson, B. J. Smith, C. M. Romagosa, J. Martin, and F. J. Mazzotti. 2017. Optimal control of an invasive species using a reaction-diffusion model and linear programming. Ecosphere 8:e01979.

Bonneau, M., J. Martin, N. Peyrard, L. Rodgers, C. M. Rornagosa, and F. A. Johnson. 2019. Optimal spatial allocation of control effort to manage invasives in the face of imperfect detection and misclassification. Ecological Modelling 392:108-116.

Caplat, P., S. Coutts, and Y. M. Buckley 2012. Modeling population dynamics, landscape structure, and management decisions for controlling the spread of invasive plants. Annals of the New York Academy of Sciences 1249:72-83.

Caplat, P., C. Hui, B. D. Maxwell, and D. A. Peltzer. 2014. Cross-scale management strategies for optimal control of trees invading from source plantations. Biological Invasions 16:677-690.

Chades, I., T. G. Martin, S. Nicol, M. A. Burgman, H. P. Possingham, and Y. M. Buckley. 2011. General rules for managing and surveying networks of pests, diseases, and endangered species. Proceedings of the National Academy of Sciences USA 108:8323-8328.

Choquenot, D., J. Hone, and G. Saunders. 1999. Using aspects of predator-prey theory to evaluate helicopter shooting for feral pig control. Wildlife Research 26:251-261.

Davis, A. J., M. B. Hooten, R. S. Miller, M. L. Farnsworth, J. Lewis, M. Moxcey, and K. M. Pepin. 2016. Inferring invasive species abundance using removal data from management actions. Ecological Applications 26:2339-2346.

Davis, A. J., B. Leland, M. Bodenchuk, K. C. VerCauteren, and K. M. Pepin. 2018a. Costs and effectiveness of damage management of an overabundant species (Sus scrofa) using aerial gunning. Wildlife Research 45:696-705.

Davis, A. J., R. McCreary, J. Psiropoulos, G. Brennan, T. Cox, A. Partin, and K. M. Pepin. 2018b. Quantifying site-level usage and certainty of absence for an invasive species through occupancy analysis of camera-trap data. Biological Invasions 20:877-890.

De Bona, S., and M. Bruneaux. 2019. Spatio-temporal dynamics of density-dependent dispersal during a population colonisation. Ecology Letters 22:634-644.

Epanchin-Niell, R. S., and A. Hastings. 2010. Controlling established invaders: integrating economics and spread dynamics to determine optimal management. Ecology Letters 13:528-541.

Epanchin-Niell, R. S., R. G. Haight, L. Berec, J. M. Kean, and A. M. Liebhold. 2012. Optimal surveillance and eradication of invasive species in heterogeneous landscapes. Ecology Letters 15:803-812.

Faubet, P., R. S. Waples, and O. E. Gaggiotti. 2007. Evaluating the performance of a multilocus Bayesian method for the estimation of migration rates. Molecular Ecology 16:11491166.

Giljohann, K. M., C. E. Hauser, N. S. G. Williams, and J. L. Moore. 2011. Optimizing invasive species control across space: willow invasion management in the Australian Alps. Journal of Applied Ecology 48:1286-1294. 
Glen, A. S., R. P. Pech, and A. E. Byrom. 2013. Connectivity and invasive species management: towards an integrated landscape approach. Biological Invasions 15:2127-2138.

Gormley, A. M., E. P. Holland, M. C. Barron, D. P. Anderson, and G. Nugent. 2016. A modelling framework for predicting the optimal balance between control and surveillance effort in the local eradication of tuberculosis in New Zealand wildlife. Preventive Veterinary Medicine 125:10-18.

Haight, R. G., and S. Polasky. 2010. Optimal control of an invasive species with imperfect information about the level of infestation. Resource and Energy Economics 32:519-533.

Hanski, I. 1998. Metapopulation dynamics. Nature 396:41-49.

Hanski, I., and O. Ovaskainen. 2000. The metapopulation capacity of a fragmented landscape. Nature 404:755-758.

Hastings, A. 2014. Persistence and management of spatially distributed populations. Population Ecology 56:21-26.

Hastings, A., and L. W. Botsford. 2006. Persistence of spatial populations depends on returning home. Proceedings of the National Academy of Sciences USA 103:6067-6072.

Haydon, D. T., et al. 2006. Low-coverage vaccination strategies for the conservation of endangered species. Nature 443:692695.

Hernandez, F. A., B. M. Parker, C. L. Pylant, T. J. Smyser, A. J. Piaggio, S. L. Lance, M. P. Milleson, J. D. Austin, and S. M. Wisely. 2018. Invasion ecology of wild pigs (Sus scrofa) in Florida, USA: the role of humans in the expansion and colonization of an invasive wild ungulate. Biological Invasions 20:1865-1880.

Holling, C. S. 1959. The components of predation as revealed by a study of small-mammal predation of the European Pine Sawfly. Canadian Entomologist 91:293-320.

Jacobi, M. N., and P. R. Jonsson. 2011. Optimal networks of nature reserves can be found through eigenvalue perturbation theory of the connectivity matrix. Ecological Applications 21:1861-1870.

Kendall, W. L., and C. T. Moore. 2012. Maximizing the utility of monitoring to the adaptive management of natural resources. Pages 74-98 in J. J. M. R. A. Gitzen, A. B. Cooper, and D. S. Licht, editors. Design and analysis of long-term ecological monitoring studies. Cambridge University Press, Cambridge, UK.

Kling, D. M., J. N. Sanchirico, and P. L. Fackler. 2017. Optimal monitoring and control under state uncertainty: application to lionfish management. Journal of Environmental Economics and Management 84:223-245.

Lowe, W. H., and F. W. Allendorf. 2010. What can genetics tell us about population connectivity? Molecular Ecology 19:3038-3051.

Mayer, J., and I. L. Brisbin, editors. 2009. Wild pigs: biology, damage, control techniques and management. Savannah River National Laboratory, Aiken, South Carolina, USA.

Meirmans, P. G. 2014. Nonconvergence in Bayesian estimation of migration rates. Molecular Ecology Resources 14:726-733.

Moore, J. L., and M. C. Runge. 2012. Combining structured decision making and value-of-information analyses to identify robust management strategies. Conservation Biology 26:810-820.
Olsson, I. C., L. A. Greenberg, E. Bergman, and K. Wysujack. 2006. Environmentally induced migration: the importance of food. Ecology Letters 9:645-651.

Pepin, K. M., A. J. Davis, F. L. Cunningham, K. C. VerCauteren, and D. C. Eckery. 2017. Potential effects of incorporating fertility control into typical culling regimes in wild pig populations. PLoS ONE 12:e0183441.

Pepin, K. M., D. W. Wolfson, R. S. Miller, M. A. Tabak, N. P. Snow, K. C. Vercauteren, and A. J. Davis. 2019. Accounting for heterogeneous invasion rates reveals management impacts on the spatial expansion of an invasive species. Ecosphere 10: e02657.

Perry, G. L. W., K. A. Moloney, and T. R. Etherington. 2017. Using network connectivity to prioritise sites for the control of invasive species. Journal of Applied Ecology 54:1238-1250.

Punt, A. E., D. S. Butterworth, C. L. de Moor, J. A. A. De Oliveira, and M. Haddon. 2016. Management strategy evaluation: best practices. Fish and Fisheries 17:303-334.

Regan, T. J., I. Chades, and H. P. Possingham. 2011. Optimally managing under imperfect detection: a method for plant invasions. Journal of Applied Ecology 48:76-85.

Rout, T. M., J. L. Moore, and M. A. McCarthy. 2014. Prevent, search or destroy? A partially observable model for invasive species management. Journal of Applied Ecology 51:804-813.

Rudnick, D. A., et al. 2012. The role of landscape connectivity in planning and implementing conservation and restoration priorities. Issues in Ecology 16:1-23.

Samarasin, P., B. J. Shuter, S. I. Wright, and F. H. Rodd. 2017. The problem of estimating recent genetic connectivity in a changing world. Conservation Biology 31:126-135.

Strevens, C. M., and M. B. Bonsall. 2011. Density-dependent population dynamics and dispersal in heterogeneous metapopulations. Journal of Animal Ecology 80:282-293.

Tabak, M. A., A. J. Piaggio, R. S. Miller, R. A. Sweitzer, and H. B. Ernest. 2017. Anthropogenic factors predict movement of an invasive species. Ecosphere 8:e01844.

Tamburello, N., B. O. Ma, and I. M. Cote. 2019. From individual movement behaviour to landscape-scale invasion dynamics and management: a case study of lionfish metapopulations. Philosophical Transactions of the Royal Society B 374:20180057.

Travis, J. M. J., and K. J. Park. 2004. Spatial structure and the control of invasive alien species. Animal Conservation 7:321330 .

van Nouhuys, S. 2016. Metapopulation ecology. eLS. John Wiley \& Sons, Chichester, UK.

Williams, J. C., C. S. ReVelle, and S. A. Levin. 2004. Using mathematical optimization models to design nature reserves. Frontiers in Ecology and the Environment 2:98-105.

Yokomizo, H., H. P. Possingham, M. B. Thomas, and Y. M. Buckley. 2009. Managing the impact of invasive species: the value of knowing the density-impact curve. Ecological Applications 19:376-386.

Younger, J. L., G. V. Clucas, D. Kao, A. D. Rogers, K. Gharbi, T. Hart, and K. J. Miller. 2017. The challenges of detecting subtle population structure and its importance for the conservation of emperor penguins. Molecular Ecology 26:3883-3897. 